\title{
CORRECTION
}

\section{Correction to: Primary vascular bone tumors in the spine: a challenge for pathologists and spine oncology surgeons}

\author{
Stefano Boriani ${ }^{1}\left[\right.$ - Riccardo Cecchinato ${ }^{1} \cdot$ Alberto Righi $^{2} \cdot$ Stefano Bandiera $^{3} \cdot$ Angelo Paolo Dei Tos $^{4,5}$. \\ Riccardo Ghermandi ${ }^{3} \cdot$ Alessandro Gasbarrini $^{3}$
}

Published online: 26 August 2019

(c) Springer-Verlag GmbH Germany, part of Springer Nature 2019

\section{Correction to: European Spine Journal (2019) 28:1502-1511 https://doi.org/10.1007/s00586-019-05930-5}

Unfortunately, the affiliation for the following authors was incorrectly published in the original publication.

Stefano Bandiera, Riccardo Ghermandi, Alessandro Gasbarrini, Alberto Righi

The complete correct affiliations are given below.

${ }^{2}$ Unit of Pathology, IRCCS Istituto Ortopedico Rizzoli, Bologna, Italy

${ }^{3}$ Unit of Oncologic and Degenerative Spine Surgery, IRCCS Istituto Ortopedico Rizzoli, Bologna, Italy

The original article can be found online at https://doi.org/10.1007/ s00586-019-05930-5.

Riccardo Cecchinato

dott.cecchinato@gmail.com

Stefano Boriani

sb@stefanoboriani.eu

1 IRCCS Istituto Ortopedico Galeazzi, Milano, Italy

2 Unit of Pathology, IRCCS Istituto Ortopedico Rizzoli, Bologna, Italy

3 Unit of Oncologic and Degenerative Spine Surgery, IRCCS Istituto Ortopedico Rizzoli, Bologna, Italy

4 Department of Pathology and Molecular Genetics, Treviso General Hospital, Treviso, Italy

5 Department of Medicine, School of Medicine, University of Padua, Padua, Italy
Publisher's Note Springer Nature remains neutral with regard to jurisdictional claims in published maps and institutional affiliations. 